\title{
Three primary malignancies in 17 years in a man with chronic hepatitis B
}

\author{
Peter D. Block ${ }^{1}$, Brianna J. Shinn¹, Christopher G. Roth ${ }^{2}$, Jeffrey P. Baliff ${ }^{3}$, Ralph G. Zinner ${ }^{4}$, Hie-Won \\ Hann $^{5}$ \\ 'Department of Medicine, Thomas Jefferson University Hospital, Philadelphia, PA 19107, USA. \\ 2Department of Radiology, Abdominal Imaging Division, Thomas Jefferson University Hospital, Philadelphia, PA 19107, USA. \\ ${ }^{3}$ Department of Pathology, Anatomy, \& Cell Biology, Thomas Jefferson University Hospital, Philadelphia, PA 19107, USA. \\ ${ }^{4}$ Department of Medical Oncology, Thomas Jefferson University Hospital, Philadelphia, PA 19107, USA. \\ ${ }^{5}$ Liver Disease prevention Center, Division of Gastroenterology and Hepatology Thomas Jefferson University Hospital, \\ Philadelphia, PA 19107, USA.
}

Correspondence to: Dr. Hie-Won Hann, FAASLD, Professor of Medicine, Liver Disease Prevention Center, Division of Gastroenterology and Hepatology, Thomas Jefferson University Hospital, Philadelphia, PA 19107, USA.

Email: hie-won.hann@jefferson.edu

\begin{abstract}
How to cite this article: Block PD, Shinn BJ, Roth CG, Baliff JP, Zinner RG, Hann HW. Three primary malignancies in 17 years in a man with chronic hepatitis B. Hepatoma Res 2019;5:21. http://dx.doi.org/10.20517/2394-5079.2019.17
\end{abstract}

Received: 6 Apr 2019 First Decision: 20 May 2019 Revised: 23 May 2019 Accepted: 28 May 2019 Published: 21 Jun 2019

Science Editor: Guang-Wen Cao Copy Editor: Cai-Hong Wang Production Editor: Jing Yu

\begin{abstract}
The occurrence of three primary malignancies in a single patient is an infrequent phenomonen with an estimated occurrence at $0.1 \%$. Notably, patients with hepatocellular carcinoma (HCC) are particularly unlikely to develop extrahepatic primary malignancies. In this light, we present a case of patient with chronic hepatitis B who developed $\mathrm{HCC}$, as well as two other primary malignancies. This case exhibits an exceedingly rare combination of cancers, underlining the importance of continued cancer surveillence in those with a history of primary malignancy.
\end{abstract}

Keywords: Hepatitis B virus, hepatocellular carcinoma, antiviral therapy, lung cancer, multiple malignancy

\section{INTRODUCTION}

Multiple primary malignancies (MPMs) is a phenomenon that has been described in the literature for nearly a century ${ }^{[1]}$. The frequency of MPMs has increased over time, which has been partly attributed to improved surveillance and treatment options for cancer. Notably, a similar uptick in cases of second primary

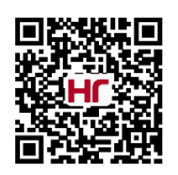


malignancies occurring in those with $\mathrm{HCC}$ has also been observed ${ }^{[2]}$. This is particularly enlightening that HCC has typically carried a grim prognosis as the third leading cause of cancer mortality worldwide ${ }^{[3]}$.

The present case report describes a patient with chronic hepatitis B, who sequentially developed bladder cancer, HCC, and lung adenocarcinoma. His complex sequence of diagnostic evaluations and therapeutic interventions are first discussed, providing context for a review of the literature on MPMs involving HCC.

\section{CASE REPORT}

A 40-year-old Asian man initially presented to an outpatient office in 1987 with a chief complaint of chronic fatigue for several months. There was no family history of malignancy or hepatitis B. He was found to be positive for the hepatitis B surface antigen $[\mathrm{HBsAg}(+)]$. He initially had an elevated alanine aminotransferase, but this returned within normal limits on repeat bloodwork. He demonstrated hepatitis B envelope antigen (HBeAg) seroconversion on follow-up labs in 1991. At the time of his diagnosis, no antiviral therapy existed, therefore he was not started on any treatment.

Nine years later, in 2000 at age 53, he developed proteinuria and hematuria and was diagnosed with bladder cancer. He underwent cystectomy with ureterostomy without complication. Three years later, he underwent cardiac angioplasty and stopped smoking cigarettes shortly thereafter. In September 2004 at age 57, abdominal imaging identified a liver mass consistent with HCC $(3.4 \mathrm{~cm}$, right lobe, segment 8$)$ with mild medial segment atrophy suggestive of underlying cirrhosis. He was started on lamivudine $150 \mathrm{mg}$ daily and then underwent transarterial chemoembolization (TACE) [Figure 1] followed by radiofrequency tumor ablation. Tenofovir $300 \mathrm{mg}$ daily was then added in May 2010. Hepatitis B virus (HBV) serology at that time demonstrated HBsAg (+), HBeAg (-), anti-HBe (+) with undetectable HBV DNA.

Despite continued antiviral therapy and undetectable HBV DNA, an abdominal MRI ten years after initial HCC [Figure 2] in 2014 revealed a new $1.0 \mathrm{~cm}$ LI-RADS 5 lesion in segment 7 consistent with recurrent HCC. At that time, liver function tests were normal, alpha-fetoprotein 2.1 and HBV DNA were still undetectable. He underwent TACE on 4/14/2014 and remained on lamivudine and tenofovir.

However, three years later, abdominal MR imaging in March 2017 revealed a $0.9 \mathrm{~cm}$ liver mass located next to the prior treatment site in segment 7. Follow-up imaging in June 2017 showed that the mass nearly doubled in size to $1.9 \mathrm{~cm}$ [Figure 3]. This prompted an evaluation for orthotopic liver transplantation (OLT). During the workup, a chest CT in August 2017 at age 70 showed a lung mass $(2.8 \mathrm{~cm} \times 2.4 \mathrm{~cm})$ in the right upper lobe [Figure 4]. A PET scan characterized the lung mass as hypermetabolic and subsequent biopsy showed mucinous adenocarcinoma of the lung. Of note, the patient had a 60 pack years smoking, stopping in 2016.

Given this new diagnosis of lung cancer, the patient was no longer eligible for OLT and therapy for both his recurrent HCC and new lung adenocarcinoma was initiated. For his HCC, he underwent TACE on early October 2017 followed by two sessions of CT-guided microwave tumor ablations. He also received five fractions of stereotactic body radiation therapy (SBRT) for his lung adenocarcinoma during this same time period. Unfortunately, surveillance PET scan in April 2018 [Figure 5] revealed a new left-sided paratracheal lymph node that was biopsy-proven mucinous adenocarcinoma representing a recurrence which was wild type for activating genetic aberrations and had a PD-L1 (by SP 263) tumor proportion score of $25 \%$. Concurrent carboplatin and paclitaxel with radiation to the mediastinum was planned. It was delayed for a month per patient request.

Concurrent chemotherapy radiation was not done as a repeat CT chest in May 2018 to establish a new baseline, which showed a new right-sided lung nodule $(1.2 \mathrm{~cm})$ [Figure 6] when biopsied was HCC. This was 

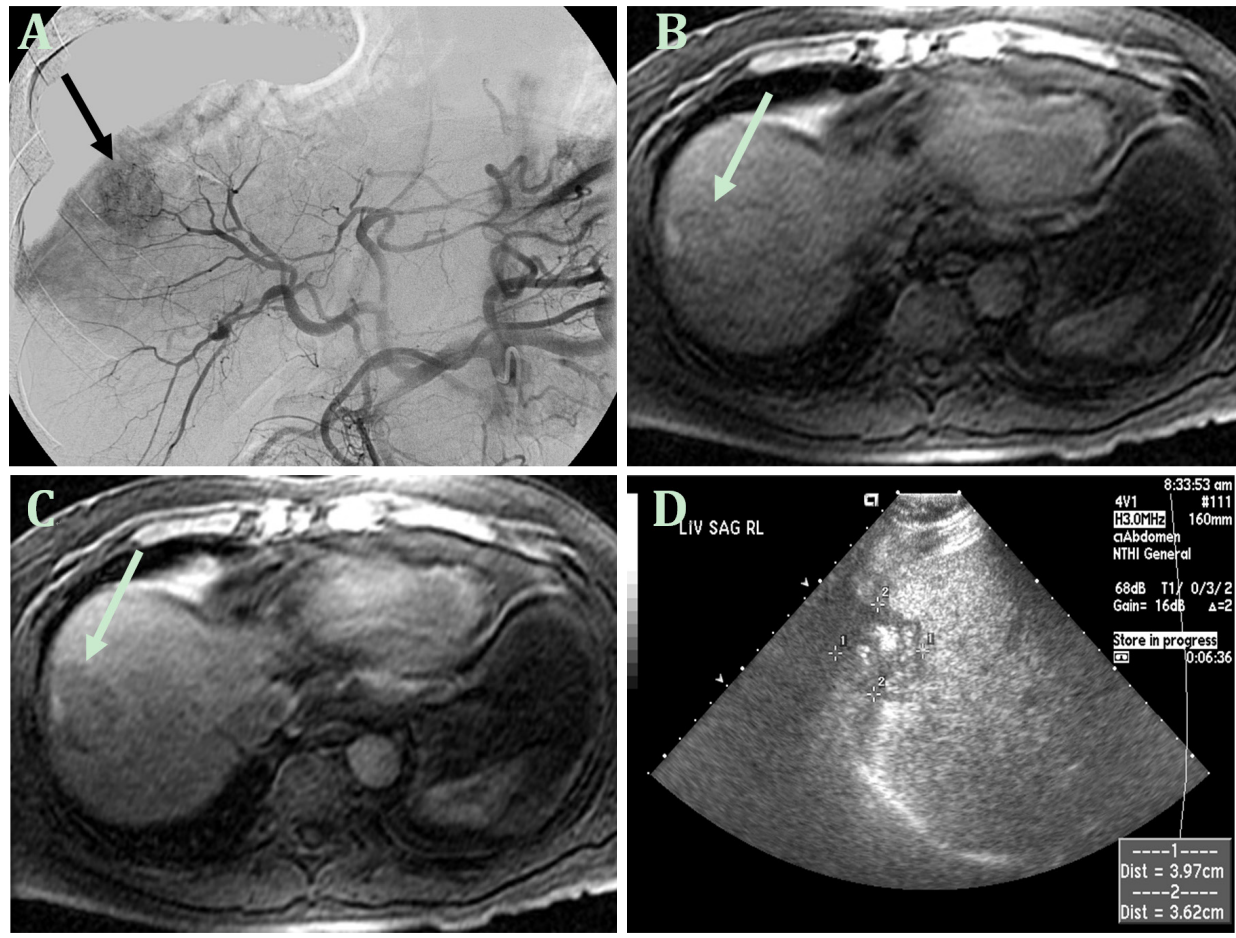

Figure 1. First diagnosis and treatment of hepatocellular carcinoma in 2004. The image from the celiac axis arteriogram in 2004 (A) shows a hyperenhancing lesion (arrow) corresponding to the hepatocellular carcinoma in segment 8; The axial T1-weighted fatsuppressed precontrast image (B) following chemoembolization shows the lesion (arrow) which fails to enhance on the subsequent postcontrast image (C); The longitudinal ultrasound image (D) shows the hypoechoic treated lesion (marked by calipers) with intralesional echogenic foci, likely reflecting posttreatment changes
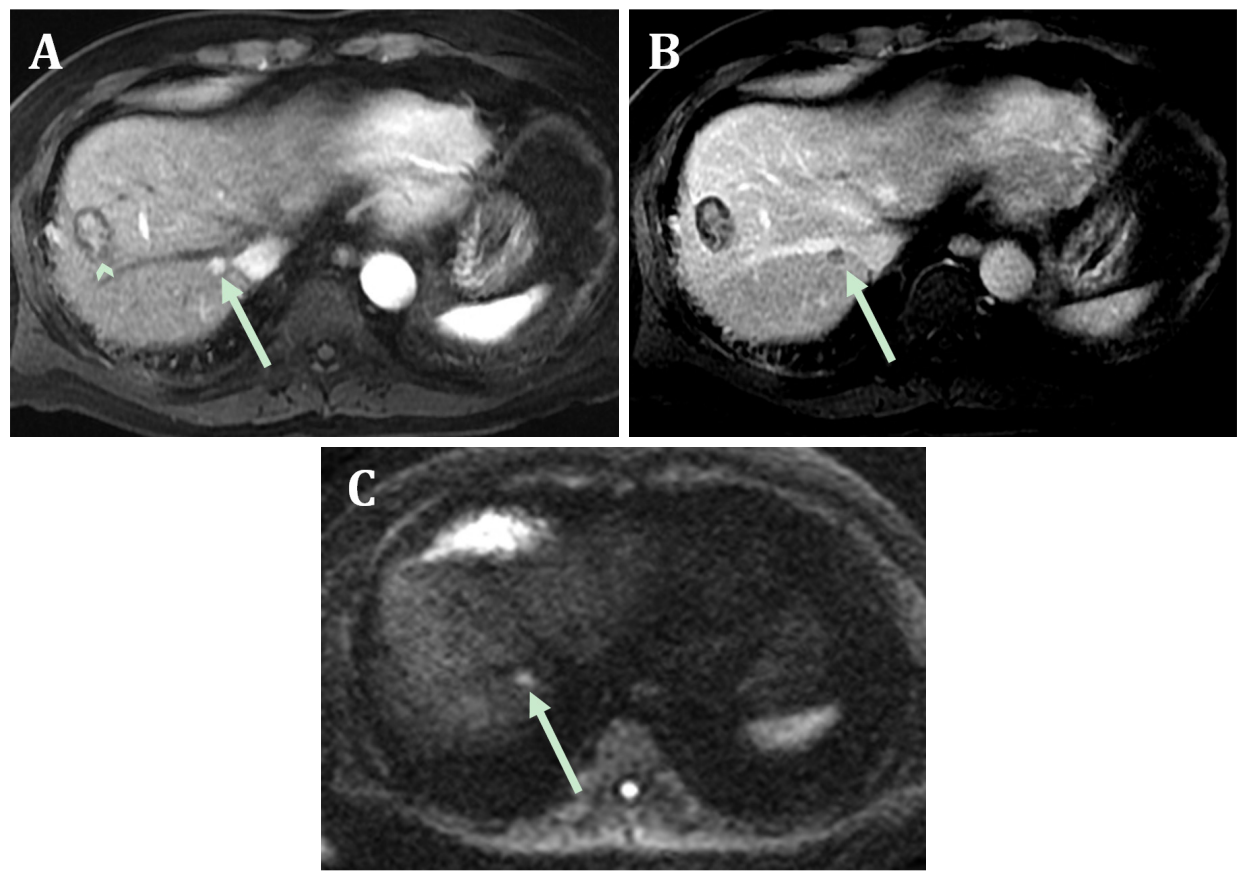

Figure 2. Recurrence of hepatocellular carcinoma on MRI in 2014. The arterial phase T1-weighted fat-suppressed postcontrast image (A) shows a small hyperenhancing lesion (arrow) in segment 7 and the previously treated lesion in segment 8 (arrowhead); The corresponding portal phase image (B) shows washout and capsule appearance and the findings conform to a LI-RADS 5 observation; the treated lesion fails to enhance (arrowhead). The diffusion-weighted image (C) reveals hyperintensity (arrow), or diffusion restriction, supporting the diagnosis of malignancy 

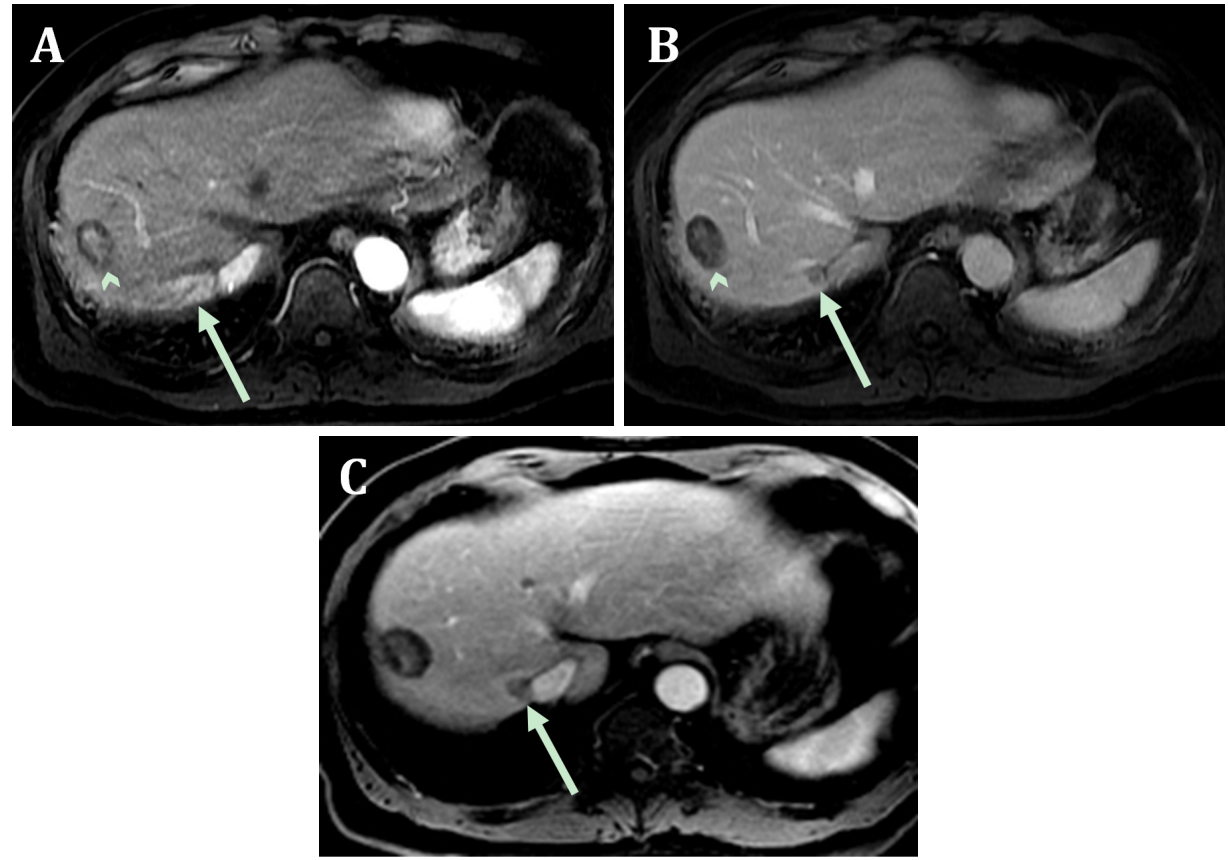

Figure 3. A second recurrence of hepatocellular carcinoma in March 2017. The arterial phase postcontrast T1-weighted, fat-suppressed image (A) shows hyperenhancement in segment 7 (arrow) adjacent to the right hepatic vein $(0.9 \mathrm{~cm}$ ); the previously treated segment 8 lesion is again noted (arrowhead). The portal phase postcontrast T1-weighted, fat-suppressed image (B) shows washout in the segment 7 lesion (arrow); the previously treated lesion (arrowhead) does not enhance. A follow-up post-contrast T1-weighted fat-suppressed three months (C) later shows an increase in lesion size to $1.9 \mathrm{~cm}$ (arrow)
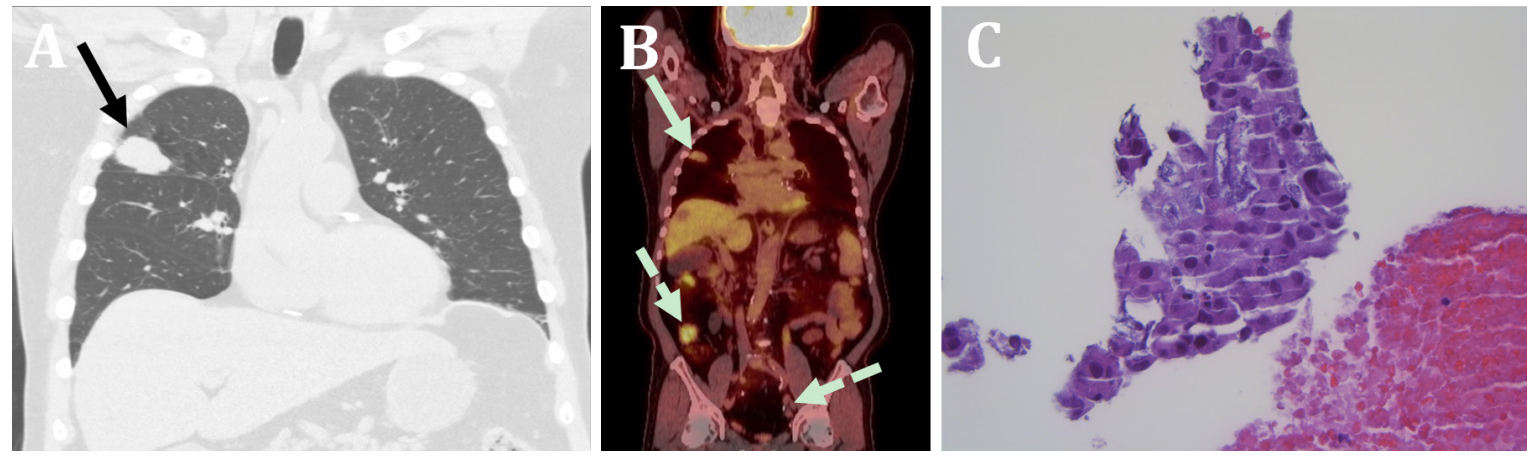

Figure 4. Diagnosis of lung adenocarcinoma in August 2017. The coronally formatted CT image (A) shows a well-circumscribed, mass-like lesion in the right upper lobe (arrow) that measures $2.8 \mathrm{~cm} \times 2.4 \mathrm{~cm}$; The image from a subsequent PET/CT (B) shows: hypermetabolism in the right upper lobe lesion (arrow) confirming malignancy; lack of metabolic activity in the previously treated segment 8 liver lesion (arrowhead) and; changes of cystectomy and right lower quadrant ileal conduit with excreted radiotracer (dashed arrows); H\&E stain (400x) of tissue from the lung mass obtained by core needle biopsy (C) show lung mucinous adenocarcinoma displaying marked cellular pleomorphism with abundant mucin production

treated with SBRT. A repeat CT chest July 2018 showed new bilateral lung nodules. A biopsy of a left lung nodule showed HCC metastasis to the lung based on positive immunohistochemical staining for hepatocyte specific antigen, Hep Par-1.

He was started on nivolumab which is active in both hepatocellular and non-small cell lung cancer manifested by the lymph nodes as above. However, after one infusion treatment, follow-up imaging [Figure 7] showed a new right hilar mass compressing the right upper bronchus. Biopsy in August 2018 showed mucinous adenocarcinoma consistent with his known lung cancer. His therapy was then switched to carboplatin, pemetrexed, and pembroluzimab of which he received four cycles with stable disease of the lung mass and 

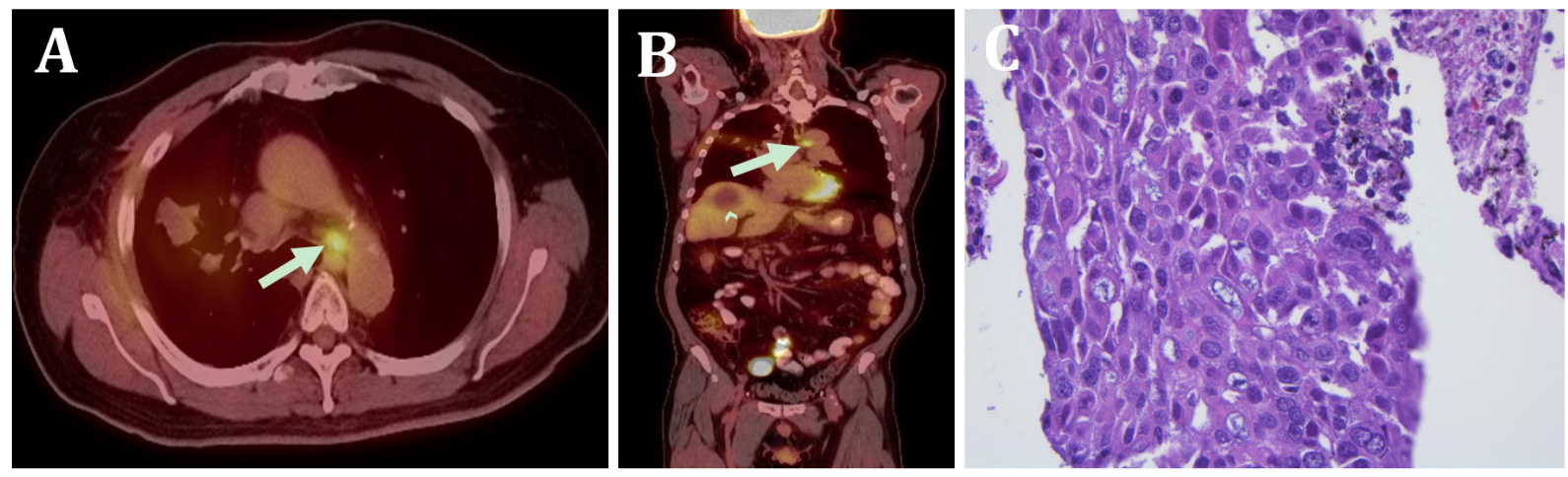

Figure 5. Metastasis of lung adenocarcinoma to paratracheal lymph node in April 2018. The axial PET/CT image (A) shows a hypermetabolic left paratracheal lymph node (arrow); The corresponding coronal PET/CT image (B) shows the hypermetabolic lymph node (arrow), as well as the treated segment 8 lesion (arrowhead); H\&E stain (400x) of the lymph tissue obtained by fine-needle aspiration (C) reveal sheets of pleomorphic cells with mucin production. This is the same morphology of lung adenocarcinoma seen on prior lung biopsy, thereby consistent with a metastasis from the lung primary tumor
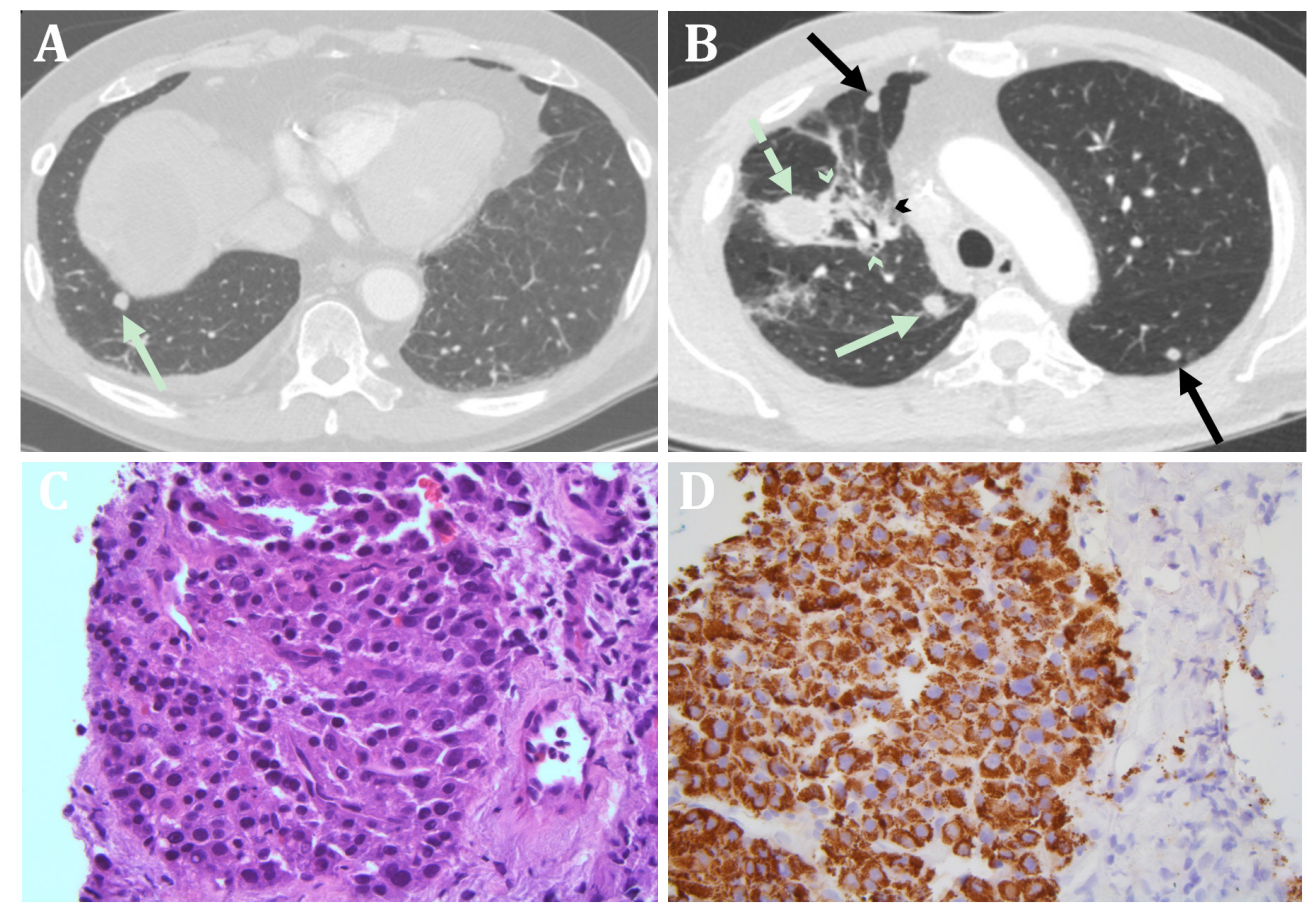

Figure 6. Metastasis of hepatocellular carcinoma to the lung in June 2018. The axial postcontrast chest CT image (A) shows the $1.2 \mathrm{~cm}$ right lower lobe nodular lesion (arrow). The axial image more superiorly shows additional nodular lesions (arrows), patchy airspace opacity (arrowheads) representing radiation treatment changes targeted to the previously noted left upper lobe mass (dashed arrow): H\&E stain (400x) of tissue from the right lower lobe lesion obtained by CT-guided core needle biopsy (C), which reveals metastatic hepatocellular carcinoma displaying a dissimilar morphology to the primary lung adenocarcinoma with relatively small and monotonous nuclei; Mucin production is also absent. Immunohistochemical stain for hepatocyte specific antigen (Hep Par-1) of the same tissue from the right lower lobe (D) shows strong positivity in tumor cells, further supporting hepatocellular origin

the bilateral lung nodules. The patient unfortunately passed away due to complications from aspiration pneumonia.

Sites and years of cancer development in the bladder, liver, lung and metastatic HCC in the lungs are shown in the diagram below. [Figure 8] 

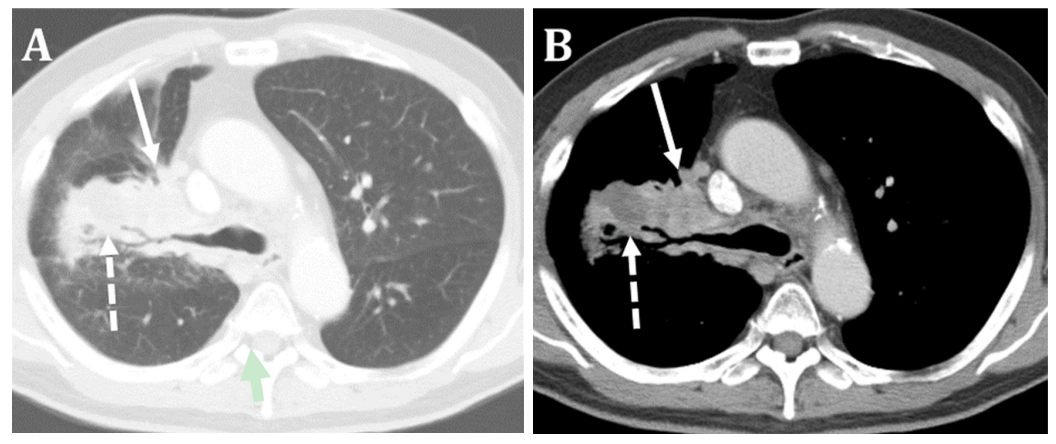

Figure 7. Progression of lung adenocarcinoma to parahilar region in August 2018. The axial postcontrast chest CT image (A) reveals the radiation scar (broken arrow) continuous with the new large right parahilar mass (solid arrow) encasing the right mainstem bronchus; The corresponding soft tissue window image (B) shows the heterogeneous enhancement of the radiation scar (broken arrow) and the right parahilar mass (solid arrow). CT-guided biopsy of this lesion (not shown) demonstrated histology compatible with metastasis of the primary lung tumor

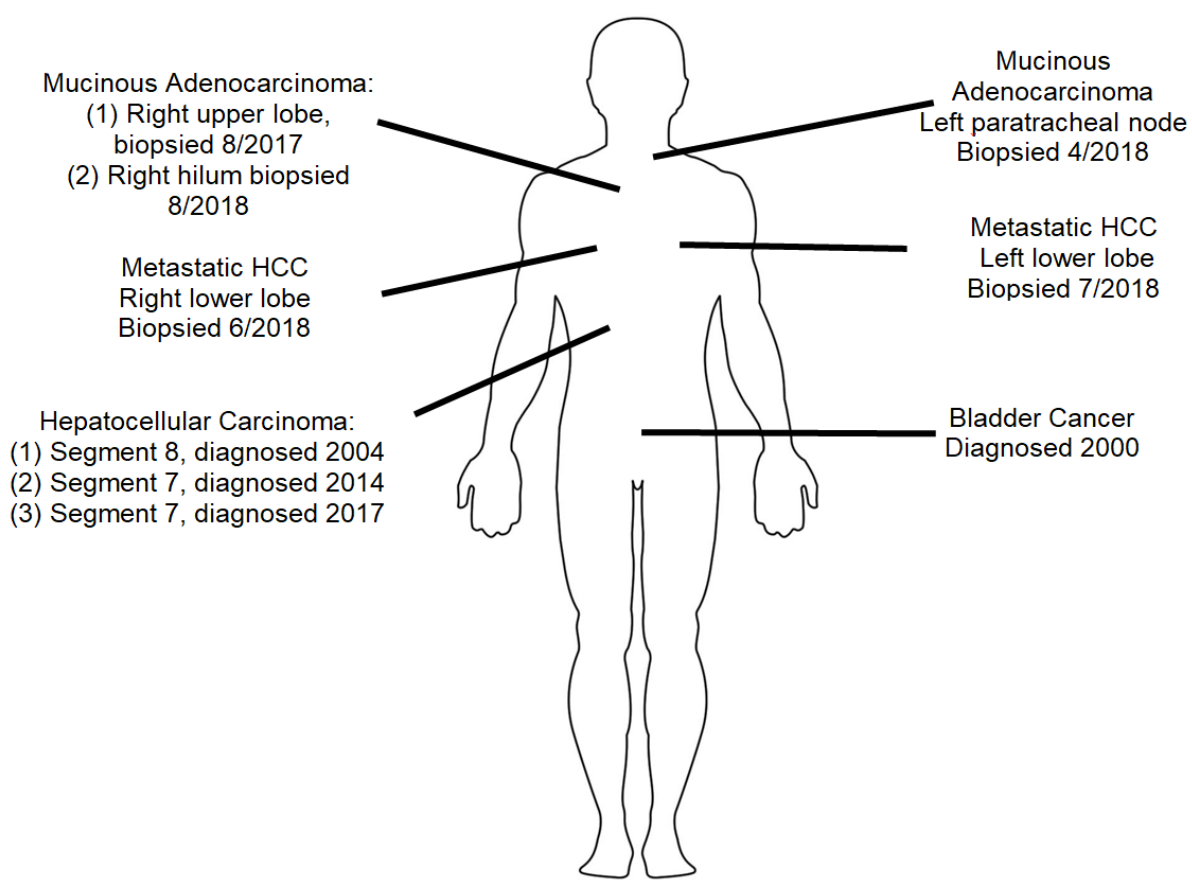

Figure 8. Site and time of cancer development in the bladder, liver, lung and HCC metastasis to the lung.

\section{DISCUSSION}

In 1932, Warren and Gates first described and defined MPMs as two or more malignant tumors of different histopathological origin in a single patient ${ }^{[1]}$. MPMs specifically in patients with HCC were initially characterized as an infrequent occurrence. This was attributed to the fact that the prognosis of HCC was historically poor. However, due to advances in screening and therapeutic options for HCC, there has been an increase in patients with HCC developing extra-hepatic primary malignancies (EHPMs) ${ }^{[2-4]}$.

This case report describes a patient who developed three primary malignancies over the span of 17 years from bladder cancer in 2000 at age 53, HCC in 2004 at age 57, and lung adenocarcinoma in 2017 at age 70. Importantly, the documented frequency of HCC occurring with two other primary malignancies in a single patient is exceedingly rare ${ }^{[2]}$. A large retrospective review of MPMs found only $57(0.1 \%)$ of the 52,398 
patients in their database had three or more primary malignancies in which none of these patients had $\mathrm{HCC}^{[5]}$. Other retrospective studies reported similar results ${ }^{[6,7]}$.

The most common sites of EHPMs in patients with HCC tend to correlate with the typical distributions of cancers found in different geographic regions. For example, studies with patient cohorts of Asian descent identified gastric and nasopharyngeal cancers as common EHPMs ${ }^{[2-4]}$, whereas studies from Western nations revealed genitourinary and colorectal cancers as the most common EHPMs ${ }^{[8]}$.

In regards to risk factors for EHPMs, studies have found similar clinical characteristics in patients with HCC who developed EHPMs. A retrospective analysis of 1506 Taiwanese patients found that in comparison to HCC patients without EHPMs, patients with HCC who developed EHPMs were more likely to be older, have earlier stage HCC, and exhibit better liver functional reserve ${ }^{[3]}$. They also found that patients with HCC and EHPMs were less likely to be chronically infected with Hepatitis B virus (HBV) or Hepatitis C virus (HCV) ${ }^{[3]}$. Retrospective studies of American, Japanese, and South Korean cohorts found similar correlations ${ }^{[4,9,10]}$.

To date, studies have been inconclusive in identifying iatrogenic or hereditary factors that may contribute to second primary malignancies in HCC patients. No clear treatment differences between HCC patients with and without EHPMs have been identified ${ }^{[2]}$. Similarly, genetic factors that may contribute to second malignancies in HCC patients have not been determined. Indeed, HCC patients with and without EHPMs have similar frequencies of family members with cancer ${ }^{[2]}$, which suggests that patterns of inheritance may be involved but these have not yet been elucidated.

In conclusion, improvements in surveillence, diagnosis, and treatment of HCC has led to an increased occurrence in the diagnosis of second primary malignancies. The case discussed here presents a rare presentation of HCC with two additional EHPMs, especially given the patient's infection with chronic HBV. Notably, these three malignancies occurred over a period of nearly two decades. This highlights the importance of continued regular cancer screening in patients with HCC, particularly those with early stage HCC with favorable prognoses. It is therefore important for physicians to be aware of EHPMs and to provide appropriate cancer screening for early detection and treatment. With this increased awareness, we hope to see an increase in overall survival in the patients with HCC and EHPMs in the future.

\section{DECLARATIONS}

\section{Authors' contributions}

Provided care for the patient with HBV and HCC, the conception and the design of the manuscript: Hann HW

Searched literature and wrote the text: Block PD, Shinn BJ

Radiological images and legends: Roth CG

Pathological examination and images: Baliff JP

Provided the lung cancer treatment: Zinner RG

\section{Availability of data and materials}

Not applicable.

\section{Financial support and sponsorship}

None.

\section{Conflicts of interest}

All authors declared that there are no conflicts of interest. 


\section{Ethical approval and consent to participate}

Not applicable.

\section{Consent for publication}

Not applicable.

\section{Copyright}

(c) The Author(s) 2019.

\section{REFERENCES}

1. Warren S, Gates O. Multiple primary malignant tumors: a survey of the literature and statistical study. Am J Cancer 1932;16:1358-414.

2. $\mathrm{Xu} \mathrm{W,} \mathrm{Liao} \mathrm{W,} \mathrm{Ge} \mathrm{P,} \mathrm{Ren} \mathrm{J,} \mathrm{Xu} \mathrm{H,} \mathrm{et} \mathrm{al.} \mathrm{Multiple} \mathrm{primary} \mathrm{malignancies} \mathrm{in} \mathrm{patients} \mathrm{with} \mathrm{hepatocellular} \mathrm{carcinoma:} \mathrm{a} \mathrm{largest} \mathrm{series} \mathrm{with}$ 26-year follow-up. Medicine (Baltimore) 2016;95:e3491.

3. Yeh ML, Huang CI, Huang CF, Hsieh MY, Lin ZY, et al. The impact of an additional extra-hepatic primary malignancy on the outcomes of patients with hepatocellular carcinoma. PLoS One 2017;12:e0184878.

4. Hong S, Jeong SH, Lee SS, Chung JW, Yang SW, et al. Prevalence and outcomes of extrahepatic primary malignancy associated with hepatocellular carcinoma in a Korean population. BMC Cancer 2015;15:146.

5. Bittorf B, Kessler H, Merkel S, Bruckl W, Wein A, et al. Multiple primary malignancies: An epidemiological and pedigree analysis of 57 patients with at least three tumours. Eur J Surg Oncol 2001;27:302-13.

6. Salem A, Abu-hijlih R, Abdelrahman F, Turfa R, Farah N, et al. Multiple primary malignancies: analysis of 23 patients with at least three tumors. J Gastrointest Cancer 2012;43:437-43.

7. Amer MH. Multiple neoplasms, single primaries, and patient survival. Cancer Manag Res 2014;6:119-34.

8. Fernandez-Ruiz M, Guerra-Vales JM, Castelbon-Fernandez FJ, Llenas-García J, Caurcel-Díaz L, et al. Multiple primary malignancies in Spanish patients with hepatocellular carcinoma: analysis of a hospital-based tumor registry. J Gastroenterol Hepatol 2009;24:1424-30.

9. Takayasu K, Kasugai H, Ikeya S, Muramatsu Y, Moriyama N, et al. A clinical and radiologic study of primary liver cancer associated with extrahepatic primary cancer. Cancer 1992;69:45-51.

10. Shah IA, Hayden CT, Alfsen GC. Association of hepatocellular carcinoma in North American patients with extrahepatic primary malignancies. Cancer 1995;75:2765-71. 